\title{
Effects of Exposure to Clothianidin on the Reproductive System of Male Quails
}

\author{
Junko TOKUMOTO ${ }^{1)}$, Megumi DANJO ${ }^{1}$, Yoshihiro KOBAYASHI $^{1)}$, Kyoko KINOSHITA ${ }^{1)}$, Takuya OMOTEHARA ${ }^{1)}$, \\ Atsutoshi TATSUMI ${ }^{1)}$, Mineo HASHIGUCHI ${ }^{3)}$, Tsuneo SEKIJIMA ${ }^{2}$, Hiroshi KAMISOYAMA $^{4)}$, \\ Toshifumi YOKOYAMA ${ }^{1)}$, Hiroshi KITAGAWA ${ }^{5)}$ and Nobuhiko HOSHI ${ }^{1) *}$ \\ 1) Laboratory of Molecular Morphology, Department of Animal Science, Graduate School of Agricultural Science, Kobe University, Kobe, \\ Hyogo 657-8501, Japan \\ 2)Environmental Science and Technology, Graduate School of Science and Technology, Niigata University, Niigata, Niigata 950-2181, \\ Japan \\ ${ }^{3)}$ Faculty of Agriculture, University Farm, Kagawa University, Sanuki, Kagawa 769-2304, Japan \\ ${ }^{4)}$ Laboratory of Animal Nutrient Metabolism, Department of Animal Science, Graduate School of Agricultural Science, Kobe University, \\ Kobe, Hyogo 657-8501, Japan \\ ${ }^{5)}$ Laboratory of Histophysiology, Department of Animal Science, Graduate School of Agricultural Science, Kobe University, Kobe, Hyogo \\ 657-8501, Japan
}

(Received 16 December 2012/Accepted 15 January 2013/Published online in J-STAGE 29 January 2013)

ABSTRACT. Clothianidin (CTD) is a neonicotinoid developed in the 1990s as an insecticide having selective toxicity, but it was later found to cause reproductive abnormalities in rats through oxidative stress. There is an attempt to preserve endangered animals, including the Japanese crested ibis, in Japan. However, there is a concern that neonicotinoid affects the reproduction of this bird, since it is used in its habitat. CTD toxicity in the birds is poorly understood, so we investigated whether or not the daily oral administration of CTD has any deleterious effects on the reproductive functions of mature male quails as experimental animals. The animals were randomly divided into four groups of 6 or 7 quails each, treated orally with 0, 0.02, 1 or $50 \mathrm{mg} \mathrm{CTD} / \mathrm{kg}$ body weight (Control, CTD0.02, CTD1 and CTD50). After that the males bred with untreated females to estimate the egg weights, and rates of fertilization and normal development, the testes, liver and spleen were examined histologically. Vacuolization and the number of germ cells having fragmented DNA in seminiferous tubules, and the number and size of vacuoles in hepatocytes increased dose-dependently. There were no significant differences in egg weights and fertilization rates between the groups, but some eggs of the CTD1 and CTD50 groups failed to develop, and embryonic length decreased dose-dependently. Thus, it was found that CTD affected the reproduction of the male quail through the fragmentation of germ cells and the inhibition or delay of embryonic development.

KEY WORDS: birds, neonicotinoid, oxidative stress, reproductive function, testis.

doi: 10.1292/jvms.12-0544; J. Vet. Med. Sci. 75(6): 755-760, 2013

Neonicotinoids, which were developed in the 1990s, are a class of neuroactive insecticides chemically related to nicotine. Until recently, they were the most widely used insecticides, because they showed reduced toxicity compared to previously used organophosphate and carbamate insecticides according to their selective toxicity. However, the use of some members of this class has been restricted in some countries due to evidence of a connection to honeybee colony collapse disorder (CCD), which led to the disappearance of over $30 \%$ of bees in America in 2007-2008 [6, 16].

In addition, several studies have reported that the breakdown products of neonicotinoids are toxic to mammals $[4,19]$, and they affect the reproductive systems of male mice [22] and rats $[2,12]$ through oxidative stress. Thus, it is suggested that neonicotinoids are harmful to mammals

\footnotetext{
*Correspondence to: Hoshi, N., Laboratory of Molecular Morphology, Department of Animal Science, Graduate School of Agricultural Science, Kobe University, 1-1 Rokkodai, Nada-ku, Kobe 657-8501, Japan.

e-mail: nobhoshi@kobe-u.ac.jp

(C)2013 The Japanese Society of Veterinary Science
}

including human.

In Japan, there is an attempt to preserve endangered animals, such as the Japanese crested ibis (Nipponia nippon). However, clothianidin (CTD), a member of neonicotinoids, is used in habitats of this bird: rice fields and farms. Since their release into the wild began in 2008, they laid eggs in the three years since, but the eggs were unfertilized. In the case of oriental stork (Ciconia boyciana), it is thought that chicks did not hatch because spermatogenesis in the male oriental stork was inhibited by residual agricultural chemicals in weatherfish [21], and in the Netherlands it is reported that many species of birds have been decreasing since the 1990s, when the use of neonicotinoid there began; indeed some species there have become extinct [17]. Thus, there is a concern that neonicotinoid also affects reproduction of birds.

In the present study, we administered CTD to mature male quails, a typical animal model of birds, daily by oral administration to investigate whether or not neonicotinoid has any deleterious effects on their reproductive functions.

\section{MATERIALS AND METHODS}

Animals: Quails were hatched and bred in our own animal facility, and 42 adults (26 males and 16 females) were used 
for this study. All quails were kept under a 14L:10D regimen and conventional conditions. They were allowed access to a diet (Mash-Neo; Toyohashi Feed Mills Co., Ltd., Toyohashi, Japan) and tap water ad libitum. This study was conducted in accordance with Japanese laws as well as with the rules and guidelines concerning the treatment and use of laboratory animals at Kobe University.

Administration: CTD (Water-soluble Dantotsu ${ }^{\circledR}$ : involving $16 \%$ of CTD; Sumitomo Chemical Co., Ltd., Tokyo, Japan), donated by Sado city (Niigata, Japan), were dissolved in water to different doses used in this study, and $500 \mu \mathrm{l} / 100 \mathrm{~g}$ of body weight dose volume was administered orally to each male quail. Twenty-six males, aged 13 weeks, were randomly divided into four groups: Control (CTD 0 $\mathrm{mg} / \mathrm{kg}$ ), CTD0.02 (CTD $0.02 \mathrm{mg} / \mathrm{kg}$ ), CTD1 (CTD $1 \mathrm{mg} /$ $\mathrm{kg}$ ), CTD50 (CTD $50 \mathrm{mg} / \mathrm{kg}$ ) and orally administered water or CTD dissolved in water daily for a period of 30 days. The dose was adjusted daily according to the body weight of each animal.

Fertility test: Three to four males (at 26 days of CTD administration) from each CTD-exposed group were mated with untreated females for 5 days to examine the fertility of the males. From the second through fifth days of breeding, we collected the eggs and incubated them. After incubation for 6 days, we opened the eggs and classified them as either unfertilized, fertilized but with undeveloped embryos or fertilized with normal embryo development egg (Fig. 1).

Sample collection: After the animals were decapitated at 17 weeks of age, the testes, liver and spleen were removed, cleared of adhering connective tissue and weighed. The relative organ weights were calculated by dividing the absolute organ weights to body weight. The left testis was fixed in $4 \%$ paraformaldehyde in phosphate buffer overnight at $4{ }^{\circ} \mathrm{C}$, dehydrated through a graded series of ethanol followed by xylene and embedded in paraffin. Then, $4-\mu \mathrm{m}-$ thick sections were cut, and each tissue section was mounted on a slide glass (Platinum; Matsunami Glass Ind., Ltd., Kishiwada, Japan) for histological and immunohistochemical analyses.

Histological and immunohistochemical analyses: For general histological analysis, the testis, liver and spleen specimens were stained with hematoxylin and eosin (HE; Merck \& Co., Inc., Whitehouse Station, NJ, U.S.A.) following the manufacturer's instructions.

After deparaffinization and hydration, the sections were immersed in absolute methanol and $0.5 \% \mathrm{H}_{2} \mathrm{O}_{2}$ for 30 min respectively at room temperature (RT) to quench the endogenous peroxidase activity. The sections were incubated with Blocking One Histo (Nacalai Tesque, Inc., Kyoto, Japan) for $1 \mathrm{hr}$ at RT for protein blocking and then were incubated with the rabbit polyclonal antibody against singlestranded DNA (ssDNA: 18731; IBL Co., Ltd., Fujioka, Japan) diluted 1:1,000 in phosphate buffered saline (PBS) for $1 \mathrm{hr}$ at RT and $18 \mathrm{hr}$ at $4^{\circ} \mathrm{C}$. After washing with PBS, the sections were reacted with goat anti-rabbit immunoglobulins conjugated to peroxidase-labeled dextran polymer in Tris$\mathrm{HCl}$ buffer (EnVision+; Dako, Glostrup, Denmark) for $1 \mathrm{hr}$ at RT. Immunoreactivity was then detected by incubation

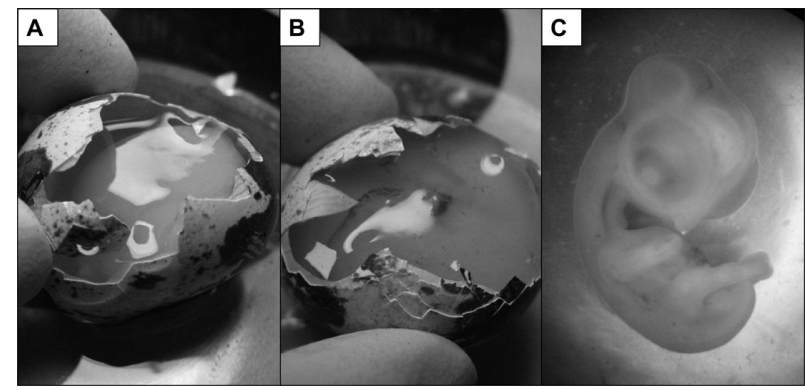

Fig. 1. Assessment of embryo development. A: unfertilized egg, B: egg whose embryonic development had stopped, C: fertilized egg.

with 3,3'-diaminobenzidine solution (EnVision+ kit/HRP [DAB], Dako). The sections were then washed with distilled water and counterstained lightly with hematoxylin solution for $1 \mathrm{~min}$. Finally, they were placed in distilled water and a graded series of ethanol, dehydrated with absolute ethanol, cleared by xylene and coverslipped with Eukitt (Sigma Chemical, St. Louis, MO, U.S.A.).

Immunohistoplanimetry: To analyze the frequency of DNA fragmented germ cells in testis, we randomly selected 20 seminiferous tubules from 5 quails each of all the groups (a total of 100 seminiferous tubules per group were examined) and counted the numbers of them located in the seminiferous tubules. Then, these numbers per seminiferous tubule of each group were calculated.

Statistical analysis: Results are presented as mean \pm SD. Values were considered statistically significant if $P<0.05$. The Tukey-Kramer and Steel-Dwass tests were used to determine differences between groups.

\section{RESULTS}

Effects of CTD on bodies and organs: Gross pathological findings. In CTD50, 4 individuals had symptoms, such as convulsions, tottering, feather ruffling and crouching. One of the 4 individuals died 8 days after treatment started. There are no symptoms or deaths in the other groups (Table 1).

Body weight gain $(B W G)$. The highest increase in BWG was detected in CTD50. BWG was significantly higher in CTD0.02 and CTD50 compared with that of Control, and in CTD50 compared with CTD0.02 ( $P<0.05$, Fig. 2A).

Relative weights of testis, liver and spleen. There were no significant differences in relative weights of testis and spleen between the groups (Fig. 2B and 2D). CTD50 showed the highest relative liver weight. The increase in weight in CTD50 was significantly greater than that in CTD1 $(P<0.05$, Fig. 2C).

Histological and immunohistological findings. Increase of vacuolization in the seminiferous epithelia and decrease of the number of germ cells were detected in a dose-dependent manner (Fig. 3). However, since sperm was detected in the lumen of seminiferous tubules in all treatment groups, we inferred that all individuals in each treatment group were 
Table 1. Gross pathological findings as treated with CTD

\begin{tabular}{lcccc}
\hline & Control & CTD0.02 & CTD1 & CTD50 \\
\hline $\mathrm{n}$ & 7 & 6 & 6 & 7 \\
Symptoms & 0 & 0 & 0 & $4^{*}$ \\
Death & 0 & 0 & 0 & $1^{* *}$ \\
\hline
\end{tabular}

*Symptoms: convulsions, tottering, feather ruffling, and crouching. **Died on 8th day of treatment. In CTD50, 4 individuals had symptoms, and 1 of them died 8 days after treatment started.

(A)

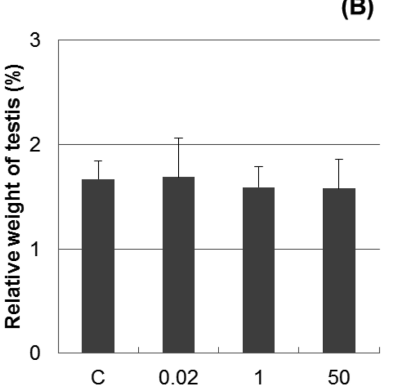

(C)

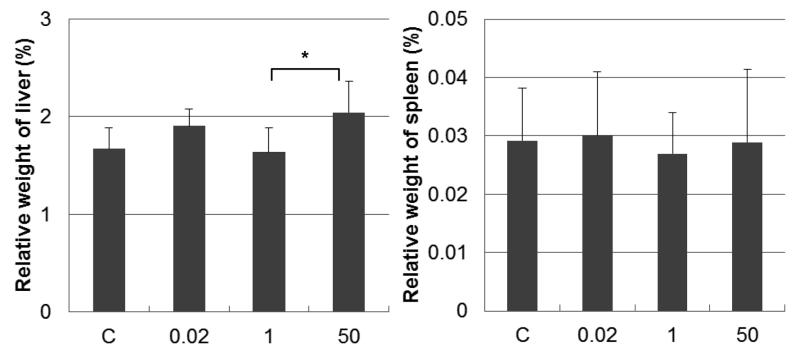

Fig. 2. Effect of treatment with CTD on body weight gain (BWG) (A), relative weights of testes (B), liver (C) and spleen (D). CTD50 shows the highest increase in BWG. CTD0.02 and CTD50 show significantly higher BWG than Control, and CTD50 has significantly higher BWG than CTD0.02 (A). There are no significant differences in relative weights of testes (B) and spleen (D) between the groups. In relative liver weights, CTD50 shows the highest level, and is significantly greater than CTD1 (C). Control: $\mathrm{n}=7$; CTD0.02, CTD1, CTD50: $\mathrm{n}=6$. Values represent the mean \pm SD. ${ }^{*}$ Significance was set at $P<0.05$.

able to produce sperm. However, the number of germ cells having fragmented DNA in seminiferous epithelia increased histologically in a dose-dependent manner (Fig. 4). Immunohistoplanimetrically, the number of ssDNA positive cells in seminiferous tubules in CTD50 was significantly larger than those in Control and CTD0.02 $(P<0.05)$, and that in CTD1 showed a tendency to be larger than that in Control $(P=0.0649$, Fig. 5). In liver, the number and size of lipid droplets in hepatocytes increased in a dose-dependent manner (Fig. 6). In spleen, there were no significant histological differences between the groups (data not shown).

Reproductive ability: Egg weight. There were no significant differences in egg weight between the groups (Fig. 7A).

Rates of fertilization and normal development. CTD50 showed the highest level of fertility among all groups,

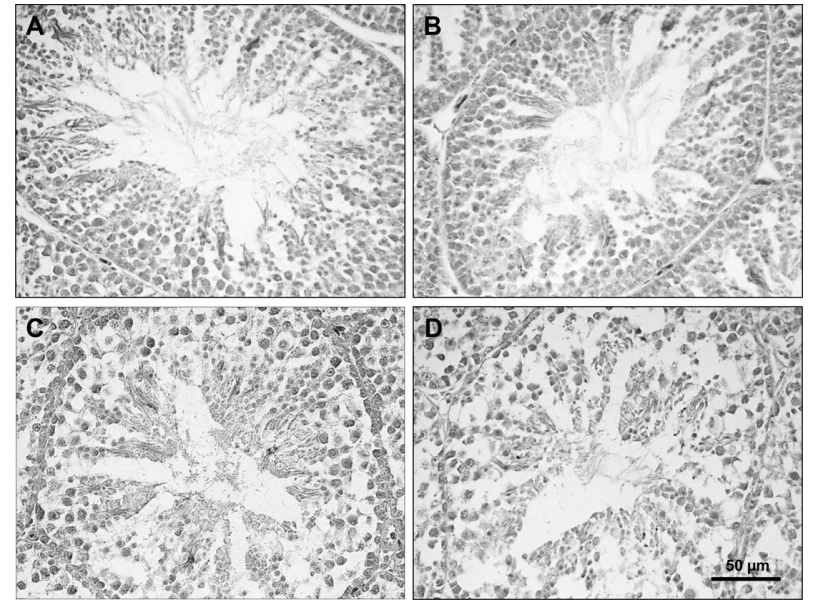

Fig. 3. Representative histology of the testis in the adult quails of Control (A), CTD0.02 (B), CTD1 (C) and CTD50 (D). The vacuolization in the seminiferous epithelia and diffusely-arranged germ cells are observed in the treatment groups (B-D) in a dosedependent manner. Sperm is seen in the lumen of seminiferous tubules in all groups. HE staining.
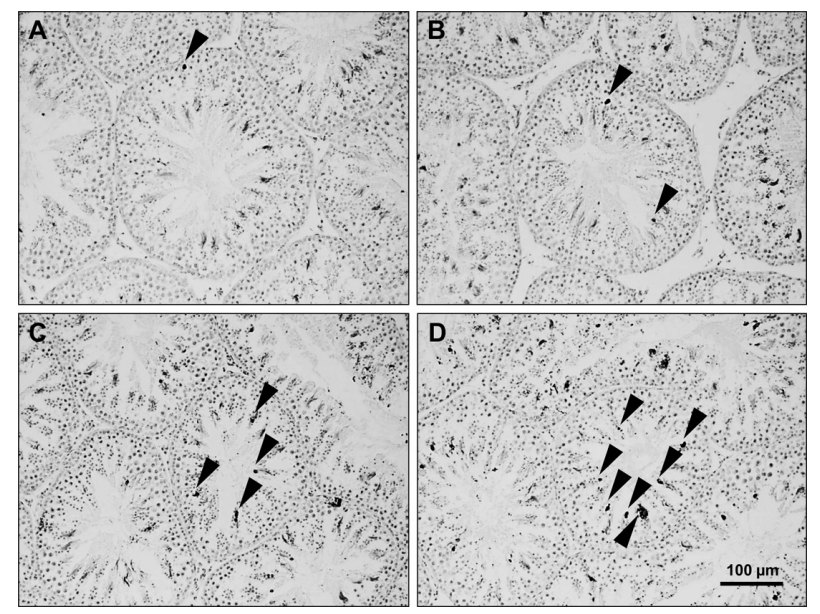

Fig. 4. The immunoreactivity for single-stranded DNA (ssDNA) is detected in the nuclei of the germ cells (arrowheads) in seminiferous epithelia of Control (A), CTD0.02 (B), CTD1 (C) and CTD50 (D). The number of immunoreactive cells increases in treatment groups (B-D) in a dose-dependent manner.

although all treated groups showed high levels (about 80\%) (Fig. 7B). However, since one egg in CTD50 and two in CTD1 stopped developing, the rates of normal development were lower than those of fertilization in CTD50 and CTD1 (Fig. 7C).

Embryo length and weight. Embryos were shorter in a dosedependent manner, and those in CTD1 were significantly shorter than the Control ( $P<0.05$, Fig. 7D). Although embryo weight did not decrease in a dose-dependent manner, that of CTD50 was the lowest among all the groups (Fig. 7E). 


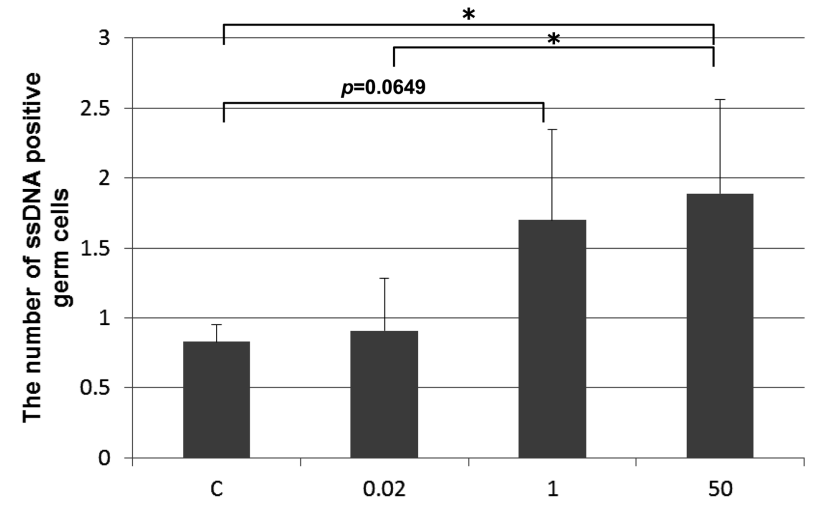

Fig. 5. The number of single-stranded DNA (ssDNA) positive germ cells in seminiferous tubules of CTD50 is significantly larger than those of Control and CTD0.02. Values represent the mean \pm SD. * Significance was set at $P<0.05$.
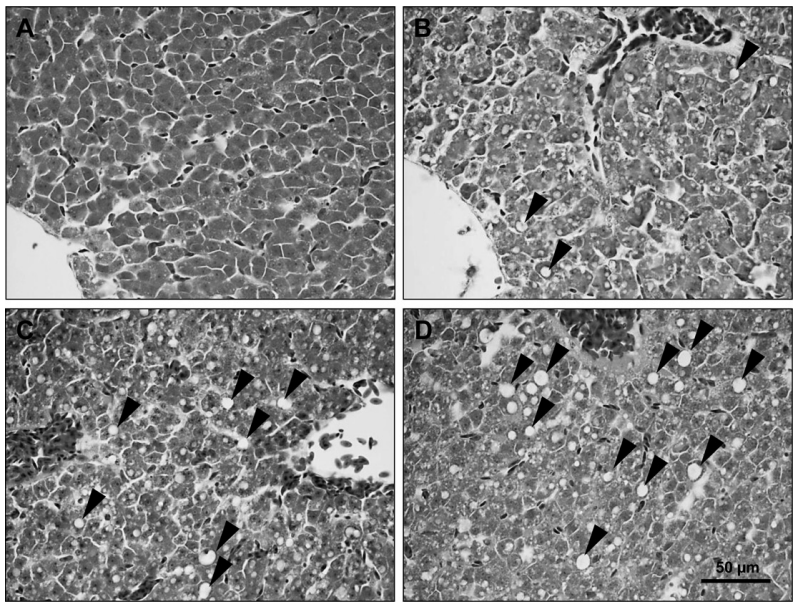

Fig. 6. Representative histology of the liver in the adult male quails of Control (A), CTD0.02 (B), CTD1 (C) and CTD50 (D). Normal hepatic lamina can be observed in the Control (A). Many vacuoles (arrowheads) and irregularly-arranged hepatocytes are seen in the treatment groups (B-D) in a dose-dependent manner. HE staining.
(A)
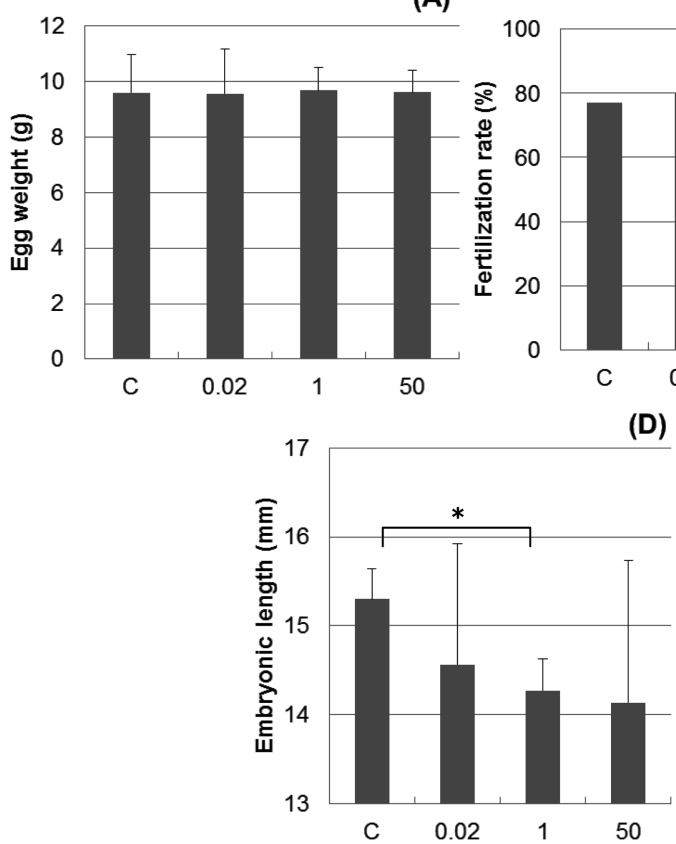

(B)

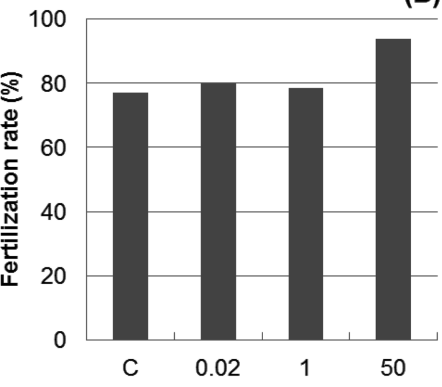

(D)

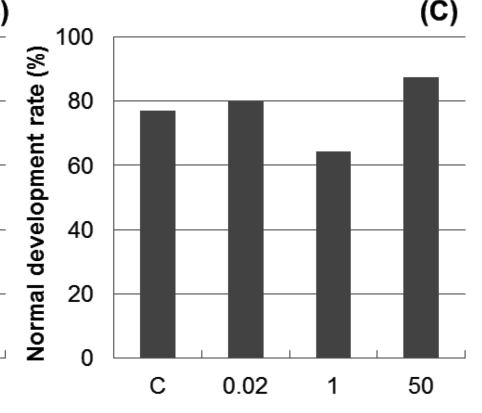

(E)

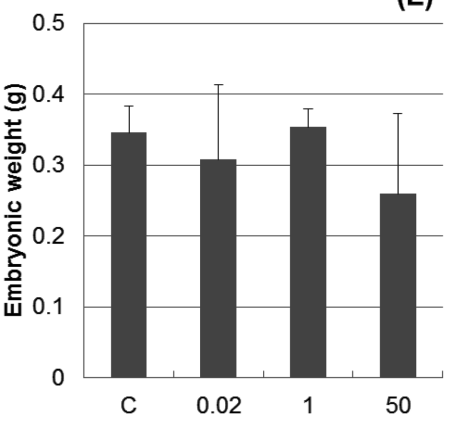

Fig. 7. Effect of treatment with CTD on egg weight (A), fertilization rate (B), normal development rate (C), embryonic length (D) and embryonic weight (E). There are no significant differences in egg weights between the groups (A). CTD50 shows the highest fertility rate among all groups, and the other groups show high rates (about $80 \%$ ) (B). The rates of normal development are lower than the fertilization rates in CTD1 and CTD50 (C). Embryo lengths in the treatment groups are lower than that in Control in a dose-dependent manner, and CTD1 embryo length differs significantly from Control (D). Although embryo weight does not decrease in a dose-dependent manner, that of CTD50 is the lowest among the groups (E). Control: $\mathrm{n}=7$; CTD0.02, CTD1, CTD50: $\mathrm{n}=6$. Values represent the mean \pm SD. * Significance was set at $P<0.05$. 


\section{DISCUSSION}

In the present study, we examined the effects of CTD, a neonicotinoid, on the reproductive system of quails. Few reports have investigated neonicotinoid's effects on reproduction in birds, so this report is thought to add important information to our understanding of the various effects of neonicotinoid.

The present results showed that, in CTD50, quails died or experienced severe symptoms as well as increased vacuolization, which was attributed to lipid droplets in the hepatocytes. Hence, the increased BWG of CTD50 may not be a healthy sign and may be the result of a decreased ability to metabolize due to the impairment of hepatic function.

Neonicotinoid, which has been reported to affect mammals through oxidative stress, reflects an imbalance between the production of reactive oxygen species (ROS) and a biological system's ability to readily detoxify the reactive intermediates or easily repair the resulting damage. Its relationship to sperm damage has been studied since 1943 [10]. Spermatozoa are particularly susceptible to oxidative stress-induced damage, because their cell membranes contain large quantities of polyunsaturated fatty acids [1], and their cytoplasm contains low concentrations of scavenging enzymes [8]. ROS can damage DNA by causing deletions, mutations and other lethal genetic effects [11]. It can also damage proteins and sugars. In these consequences, ROS is thought to cause decreasing sperm motility and to harm fertilization ability, male fertility and embryo development.

Histological changes in the liver with large areas of the degeneration of administration groups in the present study are similar to neonicotinoids administration in chickens [5] (thiacloprid), [7] (imidacloprid) and in rats [15] (thiamethoxam). Liver is a vital organ with a wide range of functions, including detoxification. Because of this function, liver is also prone to many diseases. As liver's metabolic function is activated, it produces many more ROSs, which oxidize lipids of the hepatocellular membrane, and these oxidized lipids become lipid peroxide. As a result, polysome is left out of the endoplasmic reticulum in hepatocytes, which lose the abilities to synthesize lipoprotein and to release endocytosed fatty acid; finally, lipid droplets in hepatocytes increase [14]. Since similar effects were observed in the present study, we can infer that neonicotinoid probably affects the liver through oxidative stress.

In male rats, neonicotinoid decreased sperm motility and epididymal sperm concentration, and increased the abnormal sperm rate [2]. We found that it decreased the number of germ cells and increased DNA-fragmented germ cells in seminiferous epithelium in a dose-dependent manner, but we also found normal spermatogenesis in the treatment groups and little effect on the egg weights and the fertilization rates in females bred with the treated males. Thus, it is thought that CTD does not significantly decrease reproductive function, but may affect the reproductive system potentially by decreasing germ cells by apoptosis.

The embryos from females that bred with the treated males were shorter and weighed less than embryos from Control females, or embryonic development was more likely to stop. It is reported that neonicotinoid causes DNA fragmentation of rat sperm through oxidative stress [2], and the injection of DNA-fragmented sperm into human egg cells inhibits embryo development after the eight-cell stage [18]. In addition, it is known that injury to sperm DNA after second meiosis is not repaired, and that DNA-fragmented sperm are found in human ejaculate. Although the ratios of abnormal sperm in humans and in experimental animals were reported to be 9.3-15.5\% [3, 20] and 0.9-1.4\% [9], respectively, and that of DNA-fragmented sperm in human ejaculate was reported to be $20-70 \%$ [13], the ratios of DNA-fragmented sperm in wild animals or in endangered animals may be higher than in humans or experimental animals. As the number of DNA-fragmented sperm increases, the fertilization rate of the sperm is thought to rise, so DNA fragmentation of sperm might affect embryo development. In light of this, CTD may inhibit or delay embryo development (growth retardation) by the fragmentation of sperm DNA through oxidative stress.

The present results indicate the possibility that neonicotinoid severely affects the reproductive function of quails, especially highly-susceptible individuals. Further analyses are necessary to investigate the mechanisms by which neonicotinoid leads to oxidative stress.

ACKNOWLEDGMENTS. This work was supported in part by a research grant for the study of the biodiversity of Sado city, Japan (to Tokumoto J); and by Grants-in-Aid for Scientific Research on Priority Areas (\#14042260) and for Scientific Research B (\#18380089) and C (\#19510077) from the Ministry of Education, Culture, Sports, Science and Technology of Japan (to Hoshi N).

\section{REFERENCES}

1. Alvarez, J. G. and Storey, B. T. 1995. Differential incorporation of fatty acids into and peroxidative loss of fatty acids from phospholipids of human spermatozoa. Mol. Reprod. Dev. 42: 334-346. [Medline] [CrossRef]

2. Bal, R., Türk, G., Yılmaz, Ö., Etem, E., Kuloğlu, T., Baydaş, G. and Naziroğlu, M. 2012. Effects of clothianidin exposure on sperm quality, testicular apoptosis and fatty acid composition in developing male rats. Cell Biol. Toxicol. 28: 187-200. [Medline] [CrossRef]

3. Brandriff, B., Gordon, L., Ashworth, L., Watchmaker, G., Moore, D. II., Wyrobek, A. J. and Carrano, A. V. 1985. Chromosomes of human sperm: variability among normal individuals. Hum. Genet. 70: 18-24. [Medline] [CrossRef]

4. Chao, S. L. and Casida, J. E. 1997. Interaction of imidacloprid metabolites and analogs with the nicotinic acetylcholine receptor of mouse brain in relation to toxicity. Pestic. Biochem. Physiol. 58: 77-88. [CrossRef]

5. Goyal, S., Sandhu, H. S. and Brar, R. S. 2010. Histopathological alterations induced after oral sub-acute thiacloprid toxicity in Gallus domesticus. Vet. Arh. 80: 673-682.

6. Henry, M., Béguin, M., Requier, F., Rollin, O., Odoux, J. F., Aupinel, P., Aptel, J., Tchamitchian, S. and Decourtye, A. 2012. A common pesticides decreases foraging success and survival in honey bees. Science 336: 348-350. [Medline] [CrossRef]

7. Kammon, A. M., Brar, R. S., Banga, H. S. and Sodhi, S. 2010. 
Patho-biochemical studies on hepatotoxicity and nephrotoxicity on exposure to chlorpyrifos and imidacloprid in layer chickens. Vet. Arh. 80: 663-672.

8. Kaneko, S. 2005. Human sperm preparation in ART-theory and application. J. Mamm. Ova Res. 22: 211-215. [CrossRef]

9. Kaneko, S. and Tanabe, K. 2003. The meanings and issues on sperm adjustment in ART. J. Mamm. Ova Res. 20: 47-50. [CrossRef]

10. MacLeod, J. 1943. The role of oxygen in the metabolism and motility of human spermatozoa. Am. J. Physiol. 138: 512-518.

11. Moustafa, M. H., Sharma, R. K., Thornton, J., Mascha, E., Abdel-Hafez, M. A., Thomas, A. J. Jr. and Agarwal, A. 2004. Relationship between ROS production, apoptosis and DNA denaturation in spermatozoa from patients examined for infertility. Hum. Reprod. 19: 129-138. [Medline] [CrossRef]

12. Najafi, G., Razi, M., Hoshyar, A., Shahmohamadloo, S. and Feyzi, S. 2010. The effect of chronic exposure with imidacloprid insecticide on fertility in mature male rats. Int. J. Steril. Fertil. 4: 9-16.

13. Nishida, T., Kaneko, S., Nozawa, S. and Iwamoto, T. 2001. Analysis of sperm DNA fragmentation of young men. St. Marianna Med. J. 29: 541-549.

14. Sato, H. 1999. Hepatotoxicity. pp. 111-118. In: ToxicologyLiving Organism, Environment, Ecosystem- (Fujita, S. ed.), Asakura Publishing Co., Ltd., Tokyo (in Japanese).

15. Shalaby, S. E. M., Farrag, A. R. H. and El-Saed, G. S. M. 2010.
Toxicological potential of thiamethoxam insecticide on albino rats and its residues in some organs. J. Arab Soc. Med. Res. 5: $165-172$.

16. Stokstad, E. 2012. Field research on bees raises concern about low-dose pesticides. Science 335: 1555. [Medline] [CrossRef]

17. Tennekes, H. 2011. Toxicity as function of exposure time. In: Lecture at the Institute of Public Health, University of Heidelbery, Germany.

18. Tesarik, J., Greco, E. and Mendoza, C. 2004. Late, but not early, paternal effect on human embryo development is related to sperm DNA fragmentation. Hum. Reprod. 19: 611-615. [Medline] [CrossRef]

19. Tomizawa, M. and Casida, J. E. 1999. Minor structural changes in nicotinoid insecticides confer differential subtype selectivity for mammalian nicotinic acetylcholine receptors. Br. J. Pharmacol. 127: 115-122. [Medline] [CrossRef]

20. Ueguchi, Y. and Tateno, H. 1994. Analysis of gametic chromosome. pp. 925-930. In: Obstetrical and Gynecological Practice, Kanehara \& Co., Ltd., Tokyo (in Japanese).

21. Yasuda, S. 2006. Release of oriental stork and the new point of view on dietary education. Hyogo Kyoiku 57: 1-7 (in Japanese).

22. Zhang, J. J., Wang, Y., Xiang, H. Y., Li, M. X., Li, W. H., Ma, K. G., Wang, X. Z. and Zhang, J. H. 2011. Oxidative stress: role in acetamiprid-induced impairment of the male mice reproductive system. Agric. Sci. China 10: 786-796. [CrossRef] 